\title{
Health Risk Assessment of Al and Heavy Metals in Milk Products for Different Age Groups in China
}

\author{
Meijuan Yü ${ }^{1,2}$, Yonglin $\mathrm{Liu}^{3}$, Varenyam Achal ${ }^{4}$, Qing-Long Fu ${ }^{3,5 *}$, Lanhai $\mathrm{Li}^{1,2 * *}$ \\ ${ }^{1}$ Collaborative Innovation Center of Sustainable Forestry in Southern China of Jiangsu Province, \\ Nanjing Forestry University, Nanjing, P.R. China \\ ${ }^{2}$ College of Biology and the Environment, Nanjing Forestry University, Nanjing, P.R. China \\ ${ }^{3}$ Jiangxi Institute of Analyzing and Testing, Nanchang, P.R. China \\ ${ }^{4}$ College of Resources and Environmental Science, East China Normal University, Shanghai, P.R. China \\ ${ }^{5}$ Key Laboratory of Soil Environment and Pollution Remediation, Institute of Soil Science, \\ Chinese Academy of Sciences, Nanjing, P.R. China
}

Received: September 1, 2014

Accepted: July 28, 2015

\begin{abstract}
Considering that pollutants can be transferred to the human body through food consumption, food safety is of great concern to the consumers. In order to evaluate the health risk of heavy metals to consumers by milk categories and consumer age groups via the ingestion of milk products, the contents of $\mathrm{Pb}, \mathrm{Cr}, \mathrm{Cd}, \mathrm{Cu}$, $\mathrm{Al}, \mathrm{Ni}, \mathrm{Zn}$, and Co in fermented, sterilized, and modified milk samples collected from markets in Nanchang, China, were measured by ICP-MS, and the potential health risk was assessed by the method of target hazard quotients (THQ) and hazard index (HI). Results of this study showed that the contents of all studied elements, excluding $\mathrm{Zn}$, were within the food safety limits. The mean daily exposures were estimated to be safe on comparison with the reference dose. However, total metal THQ (TTHQ) and HI values based on maximum exposure were larger than one for a baby age 0-3, which indicated that the long-term high consumption of milk products would result in high health risk for infants. The results also showed that the contribution of fermented milk to total THQ was larger than other milk products. Therefore, more attention should be paid to control the dietary intake of milk products by babies age $0-3$, and we should monitor the potential risk caused by the ingestion of fermented milk.
\end{abstract}

Keywords: milk, heavy metals, health risk assessment, food safety

\section{Introduction}

As one of the important food types, milk contains all of the macronutrients - namely protein, lipid, vitamins (A, D, and $\mathrm{B}$ groups), and minerals - that supply nutrition for the

*e-mail: fuqinglong09@mails.ucas.ac.cn

**e-mail: lilh@ms.xjb.ac.cn human body [1]. Milk and its products are the main constituents of the daily diet, especially for vulnerable groups such as infants, school-age children, and old age [2]. However, as an excretion of the mammary gland, it also contains numerous xenobiotic substances such as pesticides, disinfectants, drugs, metals, and various environmental contaminants, which constitute a technological risk factor for dairy products and consumer health [3]. 
Toxic metals in food have become a major public concern worldwide in recent decades due to their toxicity and bioaccumulation. Milk products have been demonstrated to be capable of accumulating heavy metals from diets and surrounding environment [2], which may pose a potential health threat to humans - especially children - because the incipient development in the digestive tract produces high absorption. Heavy metals, including lead $(\mathrm{Pb})$, zinc ( $\mathrm{Zn})$, copper $(\mathrm{Cu})$, and cadmium $(\mathrm{Cd})$, have been detected in milk products. Mean levels of $\mathrm{Pb}$ and $\mathrm{Cd}$ in milk products were reported to be 4.404 and $0.288 \mu \mathrm{g} / \mathrm{g}$, respectively [4]. Tona et al. reported that the samples of milk and its products in Nigeria contained different levels of $\mathrm{Pb}$ and $\mathrm{Cd}$ [5]. Pilarczyk et al. found that raw milk from an organic farm was rich in $\mathrm{Ca}$ and contained low levels of $\mathrm{Cu}$, but the $\mathrm{Pb}$ concentration was two times higher than permissible EU standards. Results of these studies indicated that milk and milk products were possibly contaminated by heavy metals [6].

It has been believed that the high exposure of these metals had the confirming negative effects on human health. For instance, ingestion of Cd-contaminated food would result in bone fractures, kidney dysfunction, and even cancer [7]. $\mathrm{Pb}$ exposure may lead to memory deterioration, prolonged reaction times, and a reduced ability to understand [8]. Additionally, copper $(\mathrm{Cu})$ and zinc $(\mathrm{Zn})$, which are considered essential micronutrients to living beings, also have non-carcinogenic hazardous effects on human health when their exposures exceed the tolerable reference doses [9, 10]. Therefore, to ensure consumer health it is necessary to determine the residual concentrations of metals in milk and evaluate their corresponding health risks.

Nanchang, the capital city of Jiangxi Province in southern China, is an important industrial city where heavy metal is one of the most important environmental issues. There have been several studies of metal contamination in food in the city [11, 12], but information concerning the heavy metal contents in milk and its products and the evaluation of their health risks is still scarce. Therefore, the major aims of this study were to determine the levels of $\mathrm{Pb}, \mathrm{Cr}, \mathrm{Cd}, \mathrm{Cu}$, $\mathrm{Al}, \mathrm{Ni}, \mathrm{Zn}$, and $\mathrm{Co}$ in fermented, sterilized, and modified milk from Nanchang, and assess their potential health risks for populations of different age groups using target health quotients (THQ) and hazard index (HI). The results of this study provide insights into heavy metal accumulation in milk and serve as a basis for comparison with other regions.

\section{Materials and Methods}

\section{Sampling}

A total of 20 samples, including five fermented milk, nine sterilized milk, and six modified milk, were randomly selected from local markets of Nanchang in January 2014. Samples were put in well-labeled, sterile, plastic containers and kept in a cooler with ice pack and then carried immediately to the laboratory for analysis.

\section{Sample Analysis}

For determination of metals, milk samples (approximately $3 \mathrm{~g}$ ) were treated by a routine dry-ashing method. Briefly, samples are dried at $200^{\circ} \mathrm{C}$ for three $\mathrm{h}$ and then incinerated in a muffle furnace at $510^{\circ} \mathrm{C}$ for two h. The sample ash was dissolved in $2.0 \mathrm{ml}$ concentered $\mathrm{HNO}_{3}$ [12]. Eight elements ( $\mathrm{Pb}, \mathrm{Cr}, \mathrm{Cd}, \mathrm{Cu}, \mathrm{Al}, \mathrm{Ni}, \mathrm{Zn}$, and $\mathrm{Co}$ ) were determined by ICP-MS (ELAN 6000, Perkin-Elmer-Sciex, USA) with an internal standard of indium. The most abundant and less interfered isotopes $\left({ }^{208} \mathrm{~Pb},{ }^{52} \mathrm{Cr},{ }^{111} \mathrm{Cd},{ }^{63} \mathrm{Cu},{ }^{27} \mathrm{Al}\right.$, ${ }^{60} \mathrm{Ni},{ }^{66} \mathrm{Zn},{ }^{59} \mathrm{Co}$, and ${ }^{115} \mathrm{In}$ ) were chosen to determine the concentration of studied elements. The coefficient of variation of replicate analyses was determined for the measurements to calculate analytical precision. Limits of Detection (LODs) were defined as three times the standard deviation of 10 runs of blank measurements as proposed by Huang et al. [14]. The LOD values of $\mathrm{Pb}, \mathrm{Cd}, \mathrm{Cu}, \mathrm{Cr}, \mathrm{Co}, \mathrm{Ni}, \mathrm{Zn}$, and Al were $2.0 \times 10^{-2}, 1.0 \times 10^{-2}, 3.0 \times 10^{-2}, 8.0 \times 10^{-2}, 1.0 \times 10^{-2}$, $2.0 \times 10^{-2}, 0.2$, and $0.32 \mathrm{ug} / \mathrm{L}$, respectively; and their recovery ratios ranged from $95.0 \%$ to $104.5 \%$. In this study, the correlation coefficients $\left(\mathrm{R}^{2}\right)$ of $\mathrm{Pb}, \mathrm{Cr}, \mathrm{Cd}, \mathrm{Cu}, \mathrm{Al}, \mathrm{Ni}, \mathrm{Zn}$, and Co were all above 0.99. All of the chemicals used in this study were of guaranteed grade. De-ionized water was used throughout the work. All experiments were conducted in duplicate and mean values were used for further calculation.

\section{Health Risk Assessment}

The non-carcinogenic health risks of a single metal via consumption of dairy products were assessed based on the THQ, a ratio of determined doses for one pollutant to its reference dose $(R f D)$, and can be calculated as the following equations (1) and (2) [15]:

$$
\begin{aligned}
& T H Q=\frac{E D I}{R f D} \\
& E D I=C \times I
\end{aligned}
$$

...where $E D I$ is the estimated daily intake of heavy metals $(\mathrm{mg} / \mathrm{kg} / \mathrm{d})$ and $R f D$ is the oral reference dose. The $R f D$ values of $\mathrm{Pb}, \mathrm{Cr}, \mathrm{Cd}, \mathrm{Cu}, \mathrm{Al}, \mathrm{Ni}, \mathrm{Zn}$, and $\mathrm{Co}$ are set to be 0.0036 [16], 1.5, 0.001, 0.04, 1.0, 0.02, 0.3, and 0.0003 $\mathrm{mg} / \mathrm{kg} / \mathrm{d}$ [17]. $C$ is the mean concentration of heavy metals in milk samples $(\mathrm{mg} / \mathrm{kg})$ and $I$ is the intake of milk per $\mathrm{kg}$ body weight per day $(\mathrm{kg} / \mathrm{day} \cdot \mathrm{bw}(\mathrm{kg}))$. If the THQ is less than one, the exposed population is unlikely to experience obvious adverse effects. The severity of health risks caused by toxic elements in foodstuffs is enhanced with the increasing THQ value.

Considering the discrepancy in body weight and daily intake of foodstuffs among different people, the health risk index was calculated according to different age groups. The average consumption amount of foodstuffs was widely used in the previous studies [12, 18]. However, in a given area, the people who consume the more foodstuffs tend to be easier at risk. Hence, this study used the average and maximum values of daily milk ingestion to represent the 
Table 1. Milk consumption data (g/day $\cdot b w)$ by age, gender group [16].

\begin{tabular}{|l|c|c|c|c|c|c|}
\hline \multirow{2}{*}{ Gender } & \multicolumn{6}{|c|}{ Age $(\mathrm{X})$} \\
\cline { 2 - 8 } & $\leq 3$ & $3<\mathrm{X} \leq 14$ & $14<\mathrm{X} \leq 20$ & $20<\mathrm{X} \leq 40$ & $40<\mathrm{X} \leq 60$ & $>60$ \\
\hline Male & $86.46(184.46)$ & $5.99(15.82)$ & $4.42(10.41)$ & $1.66(5.03)$ & $1.76(4.97)$ & $1.44(4.38)$ \\
\hline Female & $60.06(126.4)$ & $12.71(29.62)$ & $1.94(5.84)$ & $2.57(7.49)$ & $2.22(6.06)$ & $1.95(6.45)$ \\
\hline Mean & $73.26(155.43)$ & $9.35(22.72)$ & $3.18(8.13)$ & $2.12(6.26)$ & $1.99(5.51)$ & $1.70(5.42)$ \\
\hline
\end{tabular}

Numbers with brackets are maximum milk consumption, the others are the average milk consumption

dietary exposure of consumers in average and high exposure levels, respectively. According to the report of Yang et al. [19], the average and maximum values of individual milk consumption amount are represented in Table 1. And it was assumed that each fermented milk, sterilized milk, and modified milk accounted for one-third of the daily intake of milk for all age groups.

Exposure to two or more chemicals may result in additive and/or interactive effects. TTHQ has been reported to assess the overall non-carcinogenic effects of multiple heavy metals in individual foodstuffs [20]. TTHQ can be calculated as:

$$
T T H Q\left(\text { individual foodstuff) }=\sum_{i=1}^{n} T H Q_{i}\right.
$$

...where $T H Q_{i}$ is the THQ value of element $i$.

The health index, HI, was used to estimate the total nocarcinogenic health risk caused by multiple heavy metals [21]. HI for a specific receptor/pathway combination (e.g. diet) can be estimated by equation (4):

$$
H I=\sum_{i=1}^{\mathrm{n}} T T H Q_{i}
$$

...where $T T H Q_{i}$ is the TTHQ value of foodstuff $i$.

\section{Statistical Analysis}

The descriptive statistics (mean, standard deviation (SD), and range) were calculated using Microsoft Office Excel 2003. The average concentration of each heavy metal was used for further calculations. The contents of elements were log-transformed to attain or approach a normal distribution of the data. One-way analysis of variance in combination with the Duncan method was used to examine the difference of heavy metal concentrations in three types of milk products and performed by SPSS16.0. A p-value $<0.05$ was considered to indicate a significant difference between the compared groups.

\section{Results and Discussion}

\section{Concentrations of Heavy Metals in Dairy Products}

The contents of heavy metals in all analyzed milk samples and their relevant threshold values of national stan- dards were summarized in Table 2. One-way analysis of variance showed that there was no significant difference between products in terms of metal contents. However, contents of all studied elements in fermented milk were a little higher than those in other dairy products, except for $\mathrm{Cd}, \mathrm{Al}$, and $\mathrm{Ni}$, suggesting that contamination of metals may be derived from the fermentation procedure. Among all milk samples, $\mathrm{Zn}$ concentration was significantly higher than other elements $(p<0.05)$ and the overall mean contents of studied metals decreased in the order of $\mathrm{Zn}>\mathrm{Al}>\mathrm{Cu}>$ $\mathrm{Ni}>\mathrm{Cr}>\mathrm{Pb}>\mathrm{Co}>\mathrm{Cd}$. The contents of toxic heavy metals $(\mathrm{Pb}, \mathrm{Cd})$ in sterilized milk were found to be lowest among all types of milk samples (0.0062 and 0.0019 $\mathrm{mg} / \mathrm{kg}$, respectively). It was observed that $\mathrm{Pb}, \mathrm{Cr}, \mathrm{Cd}, \mathrm{Cu}$, and $\mathrm{Ni}$ in the present study were below the threshold values of standards (GB/T 2762-2012 and IDF 1979). However, Zn concentration in three modified-milk samples, three fermented-milk samples, and two sterilized-milk samples exceeded the $\mathrm{Zn}$ maximum limit of IDF 1979. The $\mathrm{Cu}$ contents in all samples were lower than the suggested normal value of $0.1 \mathrm{mg} / \mathrm{kg}$, which was in agreement with the report by Pilarczyk et al. [6]. Furthermore, the $\mathrm{Pb}$ content in the milk samples was far below its limits in both China ( 0.05 $\mathrm{mg} / \mathrm{kg})$ and the EU $(0.02 \mathrm{mg} / \mathrm{kg})$. In general, the results of this study indicate that people could safely consume milk products; nevertheless, people should pay more attention to the trace elements in milk since it is rich in $\mathrm{Zn}$ and lacks $\mathrm{Cu}$.

A comparison of heavy metal contents in this study with cow milk in other regions from various researchers is shown in Table 3. It can be seen from the table that the content of $\mathrm{Zn}$ was found to be more than the other metals, which is in accordance to other studies. Moreover, the contents of $\mathrm{Zn}, \mathrm{Cu}$, and $\mathrm{Cd}$ in this study were close to their contents in cow's milk from an organic farm in Lubuskie [6]. The levels of $\mathrm{Pb}$ and $\mathrm{Cd}$ were much lower in comparison with values observed in Egypt and Pakistan [4, 22]. The average contents of heavy metals in milk obtained from the contaminated sites proved to be larger than those from a clean area. The average contents of $\mathrm{Zn}, \mathrm{Al}, \mathrm{Ni}, \mathrm{Cu}, \mathrm{Cr}$, and Co in milk from cows kept in farms located in an industrialized region were found to be 3.163, 0.323, 0.054, 0.09, 0.088 , and $0.008 \mathrm{mg} / \mathrm{kg}$, respectively [23], which were much higher than the values of this study. Another study conducted in the vicinity of Bagjata Mine revealed that all the metals analyzed exceeded the limits [2]. There are some differences in the metals contents between this study and previous reports, which could be explained by several factors such as cow breed, local environment, and contaminat- 
Table 2. Contents of metals $(\mathrm{mg} / \mathrm{kg})$ in different types of milk.

\begin{tabular}{|c|c|c|c|c|c|c|c|}
\hline \multirow{2}{*}{ Metals } & \multicolumn{2}{|c|}{ Fermented milk $(\mathrm{n}=5)$} & \multicolumn{2}{c|}{ Sterilized milk $(\mathrm{n}=9)$} & \multicolumn{2}{c|}{ Modified milk $(\mathrm{n}=6)$} & \multirow{2}{*}{$\begin{array}{c}\text { Threshold } \\
\text { values }\end{array}$} \\
\cline { 2 - 8 } & Mean $\pm \mathrm{SD}$ & Range & Mean $\pm \mathrm{SD}$ & Range & Mean $\pm \mathrm{SD}$ & Range & $0.05^{\mathrm{a}}$ \\
\hline $\mathrm{Pb}$ & $0.0089 \pm 0.0048$ & $0.0043-0.0143$ & $0.0062 \pm 0.0022$ & $0.0036-0.0098$ & $0.0068 \pm 0.0022$ & $0.0040-0.0096$ & $0.3^{\mathrm{a}}$ \\
\hline $\mathrm{Cr}$ & $0.0087 \pm 0.0018$ & $0.0069-0.0113$ & $0.0106 \pm 0.0033$ & $0.0067-0.0185$ & $0.0085 \pm 0.001$ & $0.0064-0.0091$ & $0.026^{\mathrm{b}}$ \\
\hline $\mathrm{Cd}$ & $0.0034 \pm 0.0008$ & $0.0024-0.0043$ & $0.0019 \pm 0.0011$ & $0.0008-0.0044$ & $0.0041 \pm 0.0047$ & $0.001-0.0129$ & $0.10^{\mathrm{b}}$ \\
\hline $\mathrm{Cu}$ & $0.0621 \pm 0.0239$ & $0.0305-0.0872$ & $0.0439 \pm 0.015$ & $0.0269-0.0780$ & $0.0499 \pm 0.0106$ & $0.0347-0.0621$ & NA \\
\hline $\mathrm{Al}$ & $0.1279 \pm 0.0938$ & $0.0407-0.2609$ & $0.1728 \pm 0.0920$ & $0.0563-0.3473$ & $0.2033 \pm 0.1593$ & $0.0738-0.4397$ & $1.0^{\mathrm{a}}$ \\
\hline $\mathrm{Ni}$ & $0.0403 \pm 0.0290$ & $0.0088-0.0849$ & $0.0552 \pm 0.0813$ & $0.0201-0.2713$ & $0.0423 \pm 0.0122$ & $0.0339-0.0655$ & $3.28^{\mathrm{b}}$ \\
\hline $\mathrm{Zn}$ & $3.7055 \pm 2.3731$ & $1.2865-7.2950$ & $2.8936 \pm 0.4594$ & $2.4512-3.6495$ & $3.2456 \pm 0.7045$ & $2.2978-4.2457$ & NA \\
\hline $\mathrm{Co}$ & $0.0043 \pm 0.0025$ & $0.0014-0.0079$ & $0.0038 \pm 0.0007$ & $0.003-0.0048$ & $0.0039 \pm 0.0012$ & $0.0029-0.0061$ & N \\
\hline
\end{tabular}

${ }^{a}$ Maximum allowable levels of contaminants of food (GB/T 2762-2012), (SAC 2012) [13].

${ }^{b}$ Recorded limits of International Dairy Federation (IDF 1979) [24].

NA - Not available

Table 3. Comparisons of metal contents $(\mathrm{mg} / \mathrm{L})$ in milk products with other studies.

\begin{tabular}{|c|c|c|c|c|c|c|c|c|c|c|}
\hline Samples & Location & $\mathrm{Zn}$ & $\mathrm{Al}$ & $\mathrm{Ni}$ & $\mathrm{Cu}$ & $\mathrm{Cr}$ & $\mathrm{Pb}$ & Co & $\mathrm{Cd}$ & Reference \\
\hline Cow milk & $\begin{array}{c}\text { Bagjata mining } \\
\text { area }\end{array}$ & 4.77 & - & 0.48 & 0.56 & - & ND & - & - & {$[2]$} \\
\hline Cow milk & $\begin{array}{c}\text { Lubuskie } \\
\text { (organic farm) }\end{array}$ & 3.277 & - & - & 0.045 & - & 0.041 & - & 0.004 & [6] \\
\hline $\begin{array}{l}\text { Pasteurized } \\
\text { milk }\end{array}$ & $\begin{array}{l}\text { Arak city } \\
\text { (market) }\end{array}$ & - & 0.135 & - & - & - & 0.013 & - & 0.004 & {$[25]$} \\
\hline Cow milk & $\begin{array}{c}\text { Egypt } \\
\text { (animal farms) }\end{array}$ & 2.828 & - & 0.002 & 0.131 & 0.026 & 0.040 & 0.002 & 0.068 & {$[26]$} \\
\hline $\begin{array}{l}\text { Pasteurized } \\
\text { milk }\end{array}$ & $\begin{array}{c}\text { Italy } \\
\text { (animal farms) }\end{array}$ & 4.631 & 0.2 & - & 0.052 & - & 0.005 & - & 0 & {$[27]$} \\
\hline $\begin{array}{l}\text { Commercially } \\
\text { milk }\end{array}$ & $\begin{array}{l}\text { Munbai city } \\
\text { (market) }\end{array}$ & 3.177 & - & - & 0.043 & - & 0.002 & - & $0.7 \times 10^{-4}$ & [28] \\
\hline Cow milk & $\begin{array}{c}\text { Silesia } \\
\text { (industrialized area) }\end{array}$ & 3.163 & 0.323 & 0.054 & 0.09 & 0.088 & - & 0.008 & - & {$[23]$} \\
\hline Fermented milk & \multirow{3}{*}{$\begin{array}{l}\text { Jiangxi, China } \\
\text { (market) }\end{array}$} & 3.706 & 0.128 & 0.040 & 0.062 & 0.009 & 0.009 & 0.004 & 0.003 & \multirow{3}{*}{ This study } \\
\hline Sterilized milk & & 2.894 & 0.173 & 0.055 & 0.044 & 0.011 & 0.006 & 0.004 & 0.002 & \\
\hline Modified milk & & 3.246 & 0.203 & 0.042 & 0.05 & 0.009 & 0.007 & 0.004 & 0.004 & \\
\hline
\end{tabular}

ed feed, which could affect the metal levels in milk. In addition, production equipment, storage, and transport containers are important supplies of heavy metal contamination.

Estimated Daily Intake of Metals and Their Respective Human Health Risks

Although the contents of heavy metals in milk products were below the threshold values of standards, it cannot be concluded that there was no risk for human health. The reason is that the exposure of milk consumers to heavy metals and associated health risk is determined not only by pollutant concentrations in milk products, but also by milk consumption rates. So it is necessary to carry out health risk assessments according to the daily intake of metals. The average and maximum EDI values of heavy metals for each age group via the consumption of milk products were calculated and shown in Table 4. The trends of EDI values for metals in fermented milk, sterilized milk, and modified milk were in the order of $\mathrm{Zn}>\mathrm{Al}>\mathrm{Cu}>\mathrm{Ni}>\mathrm{Pb}>\mathrm{Cr}>$ $\mathrm{Co}>\mathrm{Cd}, \mathrm{Zn}>\mathrm{Al}>\mathrm{Ni}>\mathrm{Cu}>\mathrm{Cr}>\mathrm{Pb}>\mathrm{Co}>\mathrm{Cd}$ and $\mathrm{Zn}$ $>\mathrm{Al}>\mathrm{Cu}>\mathrm{Ni}>\mathrm{Cr}>\mathrm{Pb}>\mathrm{Cd}>\mathrm{Co}$, respectively, with intake of each metal being larger for 0-3 age group than that for other groups. This could be explained by the fact that milk products were the main food and nutrient source for infants. Hence, more attention should be paid to health risks caused by toxic metals for infants. As shown in Table 4, fermented milk was the most important source of $\mathrm{Pb}, \mathrm{Cu}, \mathrm{Zn}$, 
Table 4. Estimated daily intake (EDI) (mg/kg bw/d) of metals in milk products.

\begin{tabular}{|c|c|c|c|c|c|c|c|c|}
\hline \multirow{2}{*}{$\begin{array}{l}\text { Age group } \\
\text { (X) }\end{array}$} & \multicolumn{8}{|c|}{ EDI of heavy metals } \\
\hline & $\mathrm{Pb}$ & $\mathrm{Cr}$ & $\mathrm{Cd}$ & $\mathrm{Cu}$ & $\mathrm{Al}$ & $\mathrm{Ni}$ & $\mathrm{Zn}$ & Co \\
\hline \multicolumn{9}{|c|}{ Fermented milk } \\
\hline$\leq 3$ & $\begin{array}{c}2.18 \times 10^{-4} \\
\left(4.61 \times 10^{-4}\right)\end{array}$ & $\begin{array}{c}2.13 \times 10^{-4} \\
\left(4.53 \times 10^{-4}\right)\end{array}$ & $\begin{array}{c}8.31 \times 10^{-5} \\
\left(1.76 \times 10^{-4}\right)\end{array}$ & $\begin{array}{c}1.52 \times 10^{-3} \\
\left(3.22 \times 10^{-3}\right)\end{array}$ & $\begin{array}{c}3.12 \times 10^{-3} \\
\left(6.63 \times 10^{-3}\right)\end{array}$ & $\begin{array}{l}9.84 \times 10^{-4} \\
\left(2.09 \times 10^{-3}\right)\end{array}$ & $\begin{array}{c}0.090 \\
(0.192)\end{array}$ & $\begin{array}{c}1.04 \times 10^{-4} \\
\left(2.21 \times 10^{-4}\right)\end{array}$ \\
\hline $3<\mathrm{X} \leq 14$ & $\begin{array}{c}2.78 \times 10^{-5} \\
\left(6.74 \times 10^{-5}\right)\end{array}$ & $\begin{array}{c}2.73 \times 10^{-5} \\
\left(6.61 \times 10^{-5}\right)\end{array}$ & $\begin{array}{c}1.06 \times 10^{-5} \\
\left(2.58 \times 10^{-5}\right)\end{array}$ & $\begin{array}{c}1.94 \times 10^{-4} \\
\left(4.70 \times 10^{-4}\right)\end{array}$ & $\begin{array}{c}3.99 \times 10^{-4} \\
\left(9.68 \times 10^{-4}\right)\end{array}$ & $\begin{array}{c}1.26 \times 10^{-4} \\
\left(3.05 \times 10^{-4}\right)\end{array}$ & $\begin{array}{c}0.012 \\
(0.028)\end{array}$ & $\begin{array}{c}1.33 \times 10^{-5} \\
\left(3.22 \times 10^{-5}\right)\end{array}$ \\
\hline $14<\mathrm{X} \leq 20$ & $\begin{array}{c}9.44 \times 10^{-6} \\
\left(2.41 \times 10^{-5}\right)\end{array}$ & $\begin{array}{l}9.26 \times 10^{-6} \\
\left.2.37 \times 10^{-5}\right)\end{array}$ & $\begin{array}{c}3.61 \times 10^{-6} \\
\left(9.23 \times 10^{-6}\right)\end{array}$ & $\begin{array}{c}6.59 \times 10^{-5} \\
\left(1.68 \times 10^{-4}\right)\end{array}$ & $\begin{array}{c}1.36 \times 10^{-4} \\
\left(3.47 \times 10^{-4}\right)\end{array}$ & $\begin{array}{c}4.27 \times 10^{-5} \\
\left(1.09 \times 10^{-4}\right)\end{array}$ & $\begin{array}{c}3.93 \times 10^{-3} \\
(0.010)\end{array}$ & $\begin{array}{c}4.51 \times 10^{-6} \\
\left(1.15 \times 10^{-5}\right)\end{array}$ \\
\hline $20<\mathrm{X} \leq 40$ & $\begin{array}{c}6.32 \times 10^{-6} \\
\left(1.86 \times 10^{-5}\right)\end{array}$ & $\begin{array}{c}6.20 \times 10^{-6} \\
\left(1.83 \times 10^{-5}\right)\end{array}$ & $\begin{array}{c}2.42 \times 10^{-6} \\
\left(7.12 \times 10^{-6}\right)\end{array}$ & $\begin{array}{c}4.41 \times 10^{-5} \\
\left(1.30 \times 10^{-4}\right)\end{array}$ & $\begin{array}{c}9.08 \times 10^{-5} \\
\left(2.67 \times 10^{-4}\right)\end{array}$ & $\begin{array}{c}2.86 \times 10^{-5} \\
\left(8.42 \times 10^{-5}\right)\end{array}$ & $\begin{array}{c}2.63 \times 10^{-3} \\
\left(7.74 \times 10^{-3}\right)\end{array}$ & $\begin{array}{c}3.02 \times 10^{-6} \\
\left(8.90 \times 10^{-6}\right)\end{array}$ \\
\hline $40<\mathrm{X} \leq 60$ & $\begin{array}{c}5.97 \times 10^{-6} \\
\left(1.64 \times 10^{-5}\right)\end{array}$ & $\begin{array}{c}5.85 \times 10^{-6} \\
\left(1.61 \times 10^{-5}\right)\end{array}$ & $\begin{array}{c}2.28 \times 10^{-6} \\
\left(6.26 \times 10^{-6}\right)\end{array}$ & $\begin{array}{c}4.16 \times 10^{-5} \\
\left(1.14 \times 10^{-4}\right)\end{array}$ & $\begin{array}{c}8.57 \times 10^{-5} \\
\left(2.35 \times 10^{-4}\right)\end{array}$ & $\begin{array}{c}2.70 \times 10^{-5} \\
\left(7.42 \times 10^{-5}\right)\end{array}$ & $\begin{array}{l}2.48 \times 10^{-3} \\
\left(6.82 \times 10^{-3}\right)\end{array}$ & $\begin{array}{c}2.85 \times 10^{-6} \\
\left(7.83 \times 10^{-6}\right)\end{array}$ \\
\hline$>60$ & $\begin{array}{c}5.08 \times 10^{-6} \\
\left(1.61 \times 10^{-5}\right)\end{array}$ & $\begin{array}{c}4.98 \times 10^{-6} \\
\left(1.58 \times 10^{-5}\right)\end{array}$ & $\begin{array}{l}1.94 \times 10^{-6} \\
\left(6.16 \times 10^{-6}\right)\end{array}$ & $\begin{array}{c}3.54 \times 10^{-5} \\
\left(1.12 \times 10^{-4}\right)\end{array}$ & $\begin{array}{c}7.29 \times 10^{-5} \\
\left(2.31 \times 10^{-4}\right)\end{array}$ & $\begin{array}{c}2.30 \times 10^{-5} \\
\left(7.30 \times 10^{-5}\right)\end{array}$ & $\begin{array}{l}2.11 \times 10^{-3} \\
\left(6.71 \times 10^{-3}\right)\end{array}$ & $\begin{array}{c}2.43 \times 10^{-6} \\
\left(7.71 \times 10^{-6}\right)\end{array}$ \\
\hline \multicolumn{9}{|c|}{ Sterilized milk } \\
\hline$\leq 3$ & $\begin{array}{c}1.52 \times 10^{-4} \\
\left(3.22 \times 10^{-4}\right)\end{array}$ & $\begin{array}{c}2.6 \times 10^{-4} \\
\left(5.52 \times 10^{-4}\right)\end{array}$ & $\begin{array}{c}4.57 \times 10^{-5} \\
\left(9.70 \times 10^{-5}\right)\end{array}$ & $\begin{array}{c}1.07 \times 10^{-3} \\
\left(2.27 \times 10^{-3}\right)\end{array}$ & $\begin{array}{c}4.22 \times 10^{-3} \\
\left(8.95 \times 10^{-3}\right)\end{array}$ & $\begin{array}{c}1.35 \times 10^{-3} \\
\left(2.86 \times 10^{-3}\right)\end{array}$ & $\begin{array}{c}0.071 \\
(0.150)\end{array}$ & $\begin{array}{c}9.16 \times 10^{-5} \\
\left(1.94 \times 10^{-4}\right)\end{array}$ \\
\hline $3<\mathrm{X} \leq 14$ & $\begin{array}{c}1.94 \times 10^{-5} \\
\left(4.70 \times 10^{-5}\right)\end{array}$ & $\begin{array}{c}3.32 \times 10^{-5} \\
\left(8.06 \times 10^{-5}\right)\end{array}$ & $\begin{array}{c}5.84 \times 10^{-6} \\
\left(1.42 \times 10^{-5}\right)\end{array}$ & $\begin{array}{c}1.37 \times 10^{-4} \\
\left(3.32 \times 10^{-4}\right)\end{array}$ & $\begin{array}{c}5.39 \times 10^{-4} \\
\left(1.31 \times 10^{-3}\right)\end{array}$ & $\begin{array}{c}1.72 \times 10^{-4} \\
\left(4.18 \times 10^{-4}\right)\end{array}$ & $\begin{array}{c}9.02 \times 10^{-3} \\
(0.022)\end{array}$ & $\begin{array}{c}1.17 \times 10^{-5} \\
\left(2.84 \times 10^{-5}\right)\end{array}$ \\
\hline $14<\mathrm{X} \leq 20$ & $\begin{array}{c}6.59 \times 10^{-6} \\
\left(1.68 \times 10^{-5}\right)\end{array}$ & $\begin{array}{c}1.13 \times 10^{-5} \\
\left(2.88 \times 10^{-5}\right)\end{array}$ & $\begin{array}{c}1.98 \times 10^{-6} \\
\left(5.07 \times 10^{-6}\right)\end{array}$ & $\begin{array}{c}4.65 \times 10^{-5} \\
\left(1.19 \times 10^{-4}\right)\end{array}$ & $\begin{array}{c}1.83 \times 10^{-4} \\
\left(4.68 \times 10^{-4}\right)\end{array}$ & $\begin{array}{c}5.85 \times 10^{-5} \\
\left(1.50 \times 10^{-4}\right)\end{array}$ & $\begin{array}{c}3.07 \times 10^{-3} \\
\left(7.84 \times 10^{-3}\right)\end{array}$ & $\begin{array}{c}3.98 \times 10^{-6} \\
\left(1.02 \times 10^{-5}\right)\end{array}$ \\
\hline $20<\mathrm{X} \leq 40$ & $\begin{array}{c}4.41 \times 10^{-6} \\
\left(1.30 \times 10^{-5}\right)\end{array}$ & $\begin{array}{c}7.56 \times 10^{-6} \\
\left(2.22 \times 10^{-5}\right)\end{array}$ & $\begin{array}{c}1.33 \times 10^{-6} \\
\left(3.91 \times 10^{-6}\right)\end{array}$ & $\begin{array}{c}3.11 \times 10^{-5} \\
\left(9.17 \times 10^{-5}\right)\end{array}$ & $\begin{array}{c}1.22 \times 10^{-4} \\
\left(3.61 \times 10^{-4}\right)\end{array}$ & $\begin{array}{c}3.91 \times 10^{-5} \\
\left(1.15 \times 10^{-4}\right)\end{array}$ & $\begin{array}{c}2.05 \times 10^{-3} \\
\left(6.05 \times 10^{-3}\right)\end{array}$ & $\begin{array}{c}2.66 \times 10^{-6} \\
\left(7.84 \times 10^{-6}\right)\end{array}$ \\
\hline $40<X \leq 60$ & $\begin{array}{c}4.16 \times 10^{-6} \\
\left(1.14 \times 10^{-5}\right)\end{array}$ & $\begin{array}{c}7.13 \times 10^{-6} \\
\left(1.96 \times 10^{-5}\right)\end{array}$ & $\begin{array}{c}1.25 \times 10^{-6} \\
\left(3.44 \times 10^{-6}\right)\end{array}$ & $\begin{array}{c}2.94 \times 10^{-5} \\
\left(8.07 \times 10^{-5}\right)\end{array}$ & $\begin{array}{c}1.16 \times 10^{-4} \\
\left(3.18 \times 10^{-4}\right)\end{array}$ & $\begin{array}{c}3.70 \times 10^{-5} \\
\left(1.02 \times 10^{-4}\right)\end{array}$ & $\begin{array}{c}1.94 \times 10^{-3} \\
\left(5.32 \times 10^{-3}\right)\end{array}$ & $\begin{array}{c}2.51 \times 10^{-6} \\
\left(6.90 \times 10^{-6}\right)\end{array}$ \\
\hline$>60$ & $\begin{array}{c}3.54 \times 10^{-6} \\
\left(1.12 \times 10^{-5}\right)\end{array}$ & $\begin{array}{c}6.07 \times 10^{-6} \\
\left(1.93 \times 10^{-5}\right)\end{array}$ & $\begin{array}{c}1.07 \times 10^{-6} \\
\left(3.39 \times 10^{-6}\right)\end{array}$ & $\begin{array}{c}2.50 \times 10^{-5} \\
\left(7.94 \times 10^{-5}\right)\end{array}$ & $\begin{array}{l}9.85 \times 10^{-5} \\
\left(3.13 \times 10^{-4}\right)\end{array}$ & $\begin{array}{c}3.15 \times 10^{-5} \\
\left(9.99 \times 10^{-5}\right)\end{array}$ & $\begin{array}{c}1.65 \times 10^{-3} \\
\left(5.24 \times 10^{-3}\right)\end{array}$ & $\begin{array}{c}2.14 \times 10^{-6} \\
\left(6.79 \times 10^{-6}\right)\end{array}$ \\
\hline \multicolumn{9}{|c|}{ Modified milk } \\
\hline$\leq 3$ & $\begin{array}{c}1.67 \times 10^{-4} \\
\left(3.54 \times 10^{-4}\right)\end{array}$ & $\begin{array}{c}2.08 \times 10^{-4} \\
\left(4.41 \times 10^{-4}\right)\end{array}$ & $\begin{array}{c}1.00 \times 10^{-4} \\
\left(2.13 \times 10^{-4}\right)\end{array}$ & $\begin{array}{c}1.22 \times 10^{-3} \\
\left(2.59 \times 10^{-3}\right)\end{array}$ & $\begin{array}{c}4.96 \times 10^{-3} \\
(0.011)\end{array}$ & $\begin{array}{c}1.03 \times 10^{-3} \\
\left(2.19 \times 10^{-3}\right)\end{array}$ & $\begin{array}{c}0.079 \\
(0.168)\end{array}$ & $\begin{array}{c}9.50 \times 10^{-5} \\
\left(2.02 \times 10^{-4}\right)\end{array}$ \\
\hline $3<\mathrm{X} \leq 14$ & $\begin{array}{c}2.13 \times 10^{-5} \\
\left(5.17 \times 10^{-5}\right)\end{array}$ & $\begin{array}{c}2.65 \times 10^{-5} \\
\left(6.44 \times 10^{-5}\right)\end{array}$ & $\begin{array}{c}1.28 \times 10^{-5} \\
\left(3.11 \times 10^{-5}\right)\end{array}$ & $\begin{array}{c}1.56 \times 10^{-4} \\
\left(3.78 \times 10^{-4}\right)\end{array}$ & $\begin{array}{c}6.34 \times 10^{-4} \\
\left(1.54 \times 10^{-3}\right)\end{array}$ & $\begin{array}{c}1.32 \times 10^{-4} \\
\left(3.20 \times 10^{-4}\right)\end{array}$ & $\begin{array}{c}0.010 \\
(0.025)\end{array}$ & $\begin{array}{c}1.21 \times 10^{-5} \\
\left(2.95 \times 10^{-5}\right)\end{array}$ \\
\hline $14<\mathrm{X} \leq 20$ & $\begin{array}{c}7.24 \times 10^{-6} \\
\left(1.85 \times 10^{-5}\right)\end{array}$ & $\begin{array}{c}9.01 \times 10^{-6} \\
\left(2.30 \times 10^{-5}\right)\end{array}$ & $\begin{array}{c}4.36 \times 10^{-6} \\
\left(1.11 \times 10^{-5}\right)\end{array}$ & $\begin{array}{c}5.29 \times 10^{-5} \\
\left(1.35 \times 10^{-4}\right)\end{array}$ & $\begin{array}{c}2.15 \times 10^{-4} \\
\left(5.51 \times 10^{-4}\right)\end{array}$ & $\begin{array}{l}4.48 \times 10^{-5} \\
\left(1.15 \times 10^{-4}\right)\end{array}$ & $\begin{array}{c}3.44 \times 10^{-3} \\
\left(8.80 \times 10^{-3}\right)\end{array}$ & $\begin{array}{c}4.13 \times 10^{-6} \\
\left(1.05 \times 10^{-5}\right)\end{array}$ \\
\hline $20<\mathrm{X} \leq 40$ & $\begin{array}{c}4.85 \times 10^{-6} \\
\left(1.43 \times 10^{-5}\right)\end{array}$ & $\begin{array}{c}6.04 \times 10^{-6} \\
\left(1.78 \times 10^{-5}\right)\end{array}$ & $\begin{array}{c}2.92 \times 10^{-6} \\
\left(8.59 \times 10^{-6}\right)\end{array}$ & $\begin{array}{c}3.54 \times 10^{-5} \\
\left(1.04 \times 10^{-4}\right)\end{array}$ & $\begin{array}{c}1.44 \times 10^{-4} \\
\left(4.25 \times 10^{-4}\right)\end{array}$ & $\begin{array}{c}3.00 \times 10^{-5} \\
\left(8.84 \times 10^{-5}\right)\end{array}$ & $\begin{array}{c}2.30 \times 10^{-3} \\
\left(6.78 \times 10^{-3}\right)\end{array}$ & $\begin{array}{c}2.74 \times 10^{-6} \\
\left(8.13 \times 10^{-6}\right)\end{array}$ \\
\hline $40<X \leq 60$ & $\begin{array}{c}4.58 \times 10^{-6} \\
\left(1.26 \times 10^{-5}\right)\end{array}$ & $\begin{array}{c}5.70 \times 10^{-6} \\
\left(1.56 \times 10^{-5}\right)\end{array}$ & $\begin{array}{c}2.75 \times 10^{-6} \\
\left(7.56 \times 10^{-6}\right)\end{array}$ & $\begin{array}{l}3.34 \times 10^{-5} \\
\left(9.18 \times 10^{-5}\right)\end{array}$ & $\begin{array}{c}1.36 \times 10^{-4} \\
\left(3.74 \times 10^{-4}\right)\end{array}$ & $\begin{array}{c}2.83 \times 10^{-5} \\
\left(7.78 \times 10^{-5}\right)\end{array}$ & $\begin{array}{c}2.17 \times 10^{-3} \\
\left(5.97 \times 10^{-3}\right)\end{array}$ & $\begin{array}{c}2.61 \times 10^{-6} \\
\left(7.16 \times 10^{-6}\right)\end{array}$ \\
\hline$>60$ & $\begin{array}{c}3.90 \times 10^{-5} \\
\left(1.24 \times 10^{-5}\right)\end{array}$ & $\begin{array}{c}4.85 \times 10^{-5} \\
\left(1.54 \times 10^{-5}\right)\end{array}$ & $\begin{array}{c}2.34 \times 10^{-6} \\
\left(7.44 \times 10^{-6}\right)\end{array}$ & $\begin{array}{c}2.84 \times 10^{-5} \\
\left(9.03 \times 10^{-5}\right)\end{array}$ & $\begin{array}{c}1.16 \times 10^{-4} \\
\left(3.68 \times 10^{-4}\right)\end{array}$ & $\begin{array}{c}2.41 \times 10^{-5} \\
\left(7.65 \times 10^{-5}\right)\end{array}$ & $\begin{array}{c}1.85 \times 10^{-3} \\
\left(5.87 \times 10^{-3}\right)\end{array}$ & $\begin{array}{c}2.22 \times 10^{-6} \\
\left(7.04 \times 10^{-6}\right)\end{array}$ \\
\hline
\end{tabular}

Numbers with brackets are EDI values calculated on the basis of maximum milk consumption; the others are based on mean milk consumption. 
Table 5. THQ and TTHQ values of metals for all age groups due to milk consumption.

\begin{tabular}{|c|c|c|c|c|c|c|c|c|c|}
\hline \multirow{2}{*}{ Age (X) } & \multicolumn{8}{|c|}{ THQ } & \multirow{2}{*}{ TTHQ } \\
\hline & $\mathrm{Pb}$ & $\mathrm{Cr}$ & $\mathrm{Cd}$ & $\mathrm{Cu}$ & $\mathrm{Al}$ & $\mathrm{Ni}$ & $\mathrm{Zn}$ & Co & \\
\hline \multicolumn{10}{|c|}{ Fermented milk } \\
\hline \multirow{2}{*}{$\leq 3$} & 0.060 & $1.42 \times 10^{-4}$ & 0.083 & 0.038 & $3.12 \times 10^{-3}$ & 0.049 & 0.302 & 0.347 & 0.882 \\
\hline & $(0.128)$ & $\left(3.02 \times 10^{-4}\right)$ & $(0.176)$ & $(0.080)$ & $\left(6.63 \times 10^{-3}\right)$ & $(0.104)$ & $(0.640)$ & $(0.735)$ & $(1.872)$ \\
\hline \multirow{2}{*}{$3<X \leq 14$} & $7.72 \times 10^{-3}$ & $1.82 \times 10^{-5}$ & 0.011 & $4.85 \times 10^{-3}$ & $3.99 \times 10^{-4}$ & $6.29 \times 10^{-3}$ & 0.039 & 0.044 & 0.113 \\
\hline & $(0.019)$ & $\left(4.41 \times 10^{-5}\right)$ & $(0.026)$ & $(0.012)$ & $\left(9.68 \times 10^{-4}\right)$ & $(0.015)$ & $(0.094)$ & $(0.107)$ & $(0.273)$ \\
\hline \multirow{2}{*}{$14<X \leq 20$} & $2.62 \times 10^{-3}$ & $6.17 \times 10^{-6}$ & $3.61 \times 10^{-3}$ & $1.65 \times 10^{-3}$ & $1.36 \times 10^{-4}$ & $2.14 \times 10^{-3}$ & 0.013 & 0.015 & 0.038 \\
\hline & $\left(6.71 \times 10^{-3}\right)$ & $\left(1.58 \times 10^{-5}\right)$ & $\left(9.23 \times 10^{-3}\right)$ & $\left(4.21 \times 10^{-3}\right)$ & $\left(3.47 \times 10^{-4}\right)$ & $\left(5.46 \times 10^{-3}\right)$ & $(0.033)$ & $(0.038)$ & $(0.098)$ \\
\hline \multirow{2}{*}{$20<X \leq 40$} & $1.76 \times 10^{-3}$ & $4.13 \times 10^{-6}$ & $2.42 \times 10^{-3}$ & $1.10 \times 10^{-3}$ & $9.08 \times 10^{-5}$ & $1.43 \times 10^{-3}$ & $8.77 \times 10^{-3}$ & 0.010 & 0.026 \\
\hline & $\left(5.17 \times 10^{-3}\right)$ & $\left(1.22 \times 10^{-5}\right)$ & $\left(7.12 \times 10^{-3}\right)$ & $\left(3.25 \times 10^{-3}\right)$ & $\left(2.67 \times 10^{-4}\right)$ & $\left(4.21 \times 10^{-3}\right)$ & $(0.026)$ & $(0.030)$ & $(0.076)$ \\
\hline \multirow{2}{*}{$40<\mathrm{X} \leq 60$} & $1.66 \times 10^{-3}$ & $0.39 \times 10^{-5}$ & $2.28 \times 10^{-3}$ & $1.04 \times 10^{-3}$ & $8.57 \times 10^{-5}$ & $1.35 \times 10^{-3}$ & $8.28 \times 10^{-3}$ & $9.51 \times 10^{-3}$ & 0.024 \\
\hline & $\left(4.55 \times 10^{-3}\right)$ & $\left(1.07 \times 10^{-5}\right)$ & $\left(6.26 \times 10^{-3}\right)$ & $\left(2.86 \times 10^{-3}\right)$ & $\left(2.35 \times 10^{-4}\right)$ & $\left(3.71 \times 10^{-3}\right)$ & $(0.023)$ & $(0.026)$ & $(0.066)$ \\
\hline \multirow{2}{*}{$>60$} & $1.41 \times 10^{-3}$ & $3.32 \times 10^{-6}$ & $1.94 \times 10^{-3}$ & $8.85 \times 10^{-4}$ & $7.29 \times 10^{-5}$ & $1.15 \times 10^{-3}$ & $7.04 \times 10^{-3}$ & $8.09 \times 10^{-3}$ & 0.021 \\
\hline & $\left(4.48 \times 10^{-3}\right)$ & $\left(1.05 \times 10^{-5}\right)$ & $\left(6.16 \times 10^{-3}\right)$ & $\left(2.81 \times 10^{-3}\right)$ & $\left(2.31 \times 10^{-4}\right)$ & $\left(3.65 \times 10^{-3}\right)$ & $(0.022)$ & $(0.026)$ & $(0.065)$ \\
\hline \multicolumn{10}{|c|}{ Sterilized milk } \\
\hline \multirow{2}{*}{$\leq 3$} & 0.042 & $1.73 \times 10^{-4}$ & 0.046 & 0.027 & $4.22 \times 10^{-3}$ & 0.067 & 0.236 & 0.305 & 0.727 \\
\hline & $(0.089)$ & $\left(3.68 \times 10^{-4}\right)$ & $(0.097)$ & $(0.057)$ & $\left(8.95 \times 10^{-3}\right)$ & $(0.143)$ & $(0.50)$ & $(0.648)$ & (1.543) \\
\hline \multirow{2}{*}{$3<X \leq 14$} & $5.38 \times 10^{-3}$ & $2.21 \times 10^{-5}$ & $5.84 \times 10^{-3}$ & $3.42 \times 10^{-3}$ & $5.39 \times 10^{-4}$ & $8.60 \times 10^{-3}$ & 0.030 & 0.039 & 0.093 \\
\hline & $(0.013)$ & $\left(5.37 \times 10^{-5}\right)$ & $(0.014)$ & $\left(8.30 \times 10^{-3}\right)$ & $\left(1.31 \times 10^{-3}\right)$ & $(0.021)$ & $(0.073)$ & $(0.095)$ & $(0.225)$ \\
\hline \multirow{2}{*}{$14<\mathrm{X} \leq 20$} & $1.83 \times 10^{-3}$ & $7.52 \times 10^{-6}$ & $1.98 \times 10^{-3}$ & $1.16 \times 10^{-3}$ & $1.83 \times 10^{-4}$ & $2.92 \times 10^{-3}$ & 0.010 & 0.013 & 0.032 \\
\hline & $\left(4.68 \times 10^{-3}\right)$ & $\left(1.92 \times 10^{-5}\right)$ & $\left(5.07 \times 10^{-3}\right)$ & $\left(2.97 \times 10^{-3}\right)$ & $\left(4.68 \times 10^{-4}\right)$ & $\left(7.48 \times 10^{-3}\right)$ & $(0.026)$ & $(0.034)$ & $(0.081)$ \\
\hline \multirow{2}{*}{$20<X \leq 40$} & $1.23 \times 10^{-3}$ & $5.04 \times 10^{-6}$ & $1.33 \times 10^{-3}$ & $7.79 \times 10^{-4}$ & $1.23 \times 10^{-4}$ & $1.96 \times 10^{-3}$ & $6.85 \times 10^{-3}$ & $8.88 \times 10^{-3}$ & 0.021 \\
\hline & $\left(3.61 \times 10^{-3}\right)$ & $\left(1.48 \times 10^{-5}\right)$ & $\left(3.91 \times 10^{-3}\right)$ & $\left(2.29 \times 10^{-3}\right)$ & $\left(3.61 \times 10^{-4}\right)$ & $\left(5.77 \times 10^{-3}\right)$ & $(0.020)$ & $(0.026)$ & $(0.062)$ \\
\hline \multirow{2}{*}{$40<X \leq 60$} & $1.16 \times 10^{-3}$ & $4.75 \times 10^{-6}$ & $1.25 \times 10^{-3}$ & $7.35 \times 10^{-4}$ & $1.16 \times 10^{-4}$ & $1.85 \times 10^{-3}$ & $6.46 \times 10^{-3}$ & $8.38 \times 10^{-3}$ & 0.020 \\
\hline & $\left(3.18 \times 10^{-3}\right)$ & $\left(1.31 \times 10^{-3}\right)$ & $\left(3.44 \times 10^{-3}\right)$ & $\left(2.02 \times 10^{-3}\right)$ & $\left(3.18 \times 10^{-4}\right)$ & $\left(5.08 \times 10^{-3}\right)$ & $(0.018)$ & $(0.023)$ & $(0.055)$ \\
\hline \multirow{2}{*}{$>60$} & $9.84 \times 10^{-4}$ & $4.05 \times 10^{-6}$ & $1.07 \times 10^{-3}$ & $6.25 \times 10^{-4}$ & $9.85 \times 10^{-5}$ & $1.57 \times 10^{-3}$ & $5.50 \times 10^{-3}$ & $7.13 \times 10^{-3}$ & 0.017 \\
\hline & $\left(3.12 \times 10^{-3}\right)$ & $\left(1.28 \times 10^{-3}\right)$ & $\left(3.39 \times 10^{-3}\right)$ & $\left(1.99 \times 10^{-3}\right)$ & $\left(3.13 \times 10^{-4}\right)$ & $\left(4.99 \times 10^{-3}\right)$ & $(0.017)$ & $(0.023)$ & $(0.054)$ \\
\hline \multicolumn{10}{|c|}{ Modified milk } \\
\hline \multirow{2}{*}{$\leq 3$} & 0.046 & $1.38 \times 10^{-4}$ & 0.100 & 0.030 & $4.96 \times 10^{-3}$ & 0.052 & 0.264 & 0.317 & 0.815 \\
\hline & $(0.098)$ & $\left(2.94 \times 10^{-4}\right)$ & $(0.213)$ & $(0.065)$ & $(0.011)$ & $(0.110)$ & $(0.561)$ & $(0.672)$ & (1.729) \\
\hline \multirow{2}{*}{$3<X \leq 14$} & $5.92 \times 10^{-3}$ & $1.77 \times 10^{-5}$ & 0.013 & $3.89 \times 10^{-3}$ & $6.34 \times 10^{-4}$ & $6.60 \times 10^{-3}$ & 0.034 & 0.040 & 0.104 \\
\hline & $(0.014)$ & $\left(4.29 \times 10^{-5}\right)$ & $(0.031)$ & $\left(9.44 \times 10^{-3}\right)$ & $\left(1.54 \times 10^{-3}\right)$ & $(0.016)$ & $(0.082)$ & $(0.098)$ & $(0.253)$ \\
\hline \multirow{2}{*}{$14<X \leq 20$} & $2.01 \times 10^{-3}$ & $6.01 \times 10^{-6}$ & $4.36 \times 10^{-3}$ & $1.32 \times 10^{-3}$ & $2.15 \times 10^{-4}$ & $2.24 \times 10^{-3}$ & 0.011 & 0.014 & 0.035 \\
\hline & $\left(5.14 \times 10^{-3}\right)$ & $\left(1.54 \times 10^{-5}\right)$ & $(0.011)$ & $\left(3.38 \times 10^{-3}\right)$ & $\left(5.51 \times 10^{-4}\right)$ & $\left(5.73 \times 10^{-3}\right)$ & $(0.029)$ & $(0.035)$ & $(0.090)$ \\
\hline \multirow{2}{*}{$20<\mathrm{X} \leq 40$} & $1.35 \times 10^{-3}$ & $4.02 \times 10^{-6}$ & $2.92 \times 10^{-3}$ & $8.86 \times 10^{-4}$ & $1.44 \times 10^{-4}$ & $1.50 \times 10^{-3}$ & $7.68 \times 10^{-3}$ & $9.21 \times 10^{-3}$ & 0.024 \\
\hline & $\left(3.97 \times 10^{-3}\right)$ & $\left(1.18 \times 10^{-5}\right)$ & $\left(8.59 \times 10^{-3}\right)$ & $\left(2.61 \times 10^{-3}\right)$ & $\left(4.25 \times 10^{-4}\right)$ & $\left(4.42 \times 10^{-3}\right)$ & $(0.023)$ & $(0.027)$ & $(0.070)$ \\
\hline \multirow{2}{*}{$40<\mathrm{X} \leq 60$} & $1.27 \times 10^{-3}$ & $3.80 \times 10^{-6}$ & $2.75 \times 10^{-3}$ & $8.36 \times 10^{-4}$ & $1.36 \times 10^{-4}$ & $1.42 \times 10^{-3}$ & $7.25 \times 10^{-3}$ & $8.69 \times 10^{-3}$ & 0.022 \\
\hline & $\left(3.49 \times 10^{-3}\right)$ & $\left(1.04 \times 10^{-5}\right)$ & $\left(7.56 \times 10^{-3}\right)$ & $\left(2.30 \times 10^{-3}\right)$ & $\left(3,74 \times 10^{-4}\right)$ & $\left(3.89 \times 10^{-3}\right)$ & $(0.020)$ & $(0.024)$ & $(0.061)$ \\
\hline \multirow{2}{*}{$>60$} & $1.08 \times 10^{-3}$ & $3.23 \times 10^{-6}$ & $2.34 \times 10^{-3}$ & $7.11 \times 10^{-4}$ & $1.16 \times 10^{-4}$ & $1.21 \times 10^{-3}$ & $6.17 \times 10^{-3}$ & $7.39 \times 10^{-3}$ & 0.019 \\
\hline & $\left(3.44 \times 10^{-3}\right)$ & $\left(1.03 \times 10^{-5}\right)$ & $\left(7.44 \times 10^{-3}\right)$ & $\left(2.26 \times 10^{-3}\right)$ & $\left(3.68 \times 10^{-4}\right)$ & $\left(3.83 \times 10^{-3}\right)$ & $(0.02)$ & $(0.023)$ & $(0.060)$ \\
\hline
\end{tabular}

Numbers with brackets are calculated on the basis of maximum milk exposure; the others are based on average milk exposure. 
Table 6. HI for all age groups via ingestion of three milk products.

\begin{tabular}{|l|c|c|c|c|c|c|}
\hline \multirow{2}{*}{\multicolumn{1}{c|}{ HI }} & \multicolumn{7}{c|}{ Age $(\mathrm{X})$} \\
\cline { 2 - 8 } & $\leq 3$ & $3<\mathrm{X} \leq 14$ & $14<\mathrm{X} \leq 20$ & $20<\mathrm{X} \leq 40$ & $40<\mathrm{X} \leq 60$ & $>60$ \\
\hline Mean exposure & 2.424 & 0.310 & 0.105 & 0.070 & 0.067 & 0.057 \\
\hline Maximum exposure & 5.144 & 0.752 & 0.269 & 0.207 & 0.183 & 0.180 \\
\hline
\end{tabular}

and Co for all age groups, followed by modified milk, and then sterilized milk. The largest contributor of studied elements to daily intake is $\mathrm{Zn}$, which accounted for more than $90 \%$ of total heavy metals intake.

THQ has been recognized as a useful parameter for evaluating health risks associated with the consumption of metalcontaminated food crops [29]. As shown in Table 5, for both the average and maximum consumption of milk products, no THQ value over 1 was observed for all age groups, which indicated that people would not experience a significant health risk if the assessment was conducted on the basis of individual heavy metals from dairy products. It was noticed that for young children under 3 years of age, the maximum values of THQ for $\mathrm{Zn}$ and Co were closer to one, implying that $\mathrm{Co}$ and $\mathrm{Zn}$ were of concern for kids in terms of non-cancer risk. The observation that health risk was found to decrease with the increase of age may result from differences in milk consumption. In addition, the health risk level of $\mathrm{Cd}$ was higher than that of $\mathrm{Pb}$, which was in agreement with a previous report [30]. Results of this study indicated that $\mathrm{Co}$ was the major contributor to total THQ from milk in each age group, accounting for $39 \%$ of fermented milk, $42 \%$ of sterilized milk, and $38 \%$ of modified milk, followed by $\mathrm{Zn}$, which accounted for $34 \%, 32 \%$, and $32 \%$ for fermented, sterilized, and modified milk products, respectively.

Risk assessments of individual metals via milk ingestion were found to be within safe limits $(\mathrm{THQ}<1)$; whereas exposure to multiple heavy metals could result in additive effects. Therefore, the TTHQ values (sum of individual metal THQ) were calculated in this study. As shown in Table 5, for average exposure, the data of TTHQ for all age groups were less than one, which indicated that there was no potential health risk to general people by consumption of individual milk products. However, the TTHQ based on the maximum exposure level had values greater than one for the 0-3 age group, suggesting that these people - especially those consuming more milk products over a long period of time - may experience some adverse health effects. The TTHQ for each age group decreased in the order of fermented milk $>$ modified milk $>$ sterilized milk. Their corresponding contributions to total THQs were $36.39 \%$, $33.60 \%$, and $29.99 \%$ for infants age $0-3$, implying that fermented milk was the major source contributing to potential health risk.

The above evaluation was based on the ingestion of individual foodstuffs. Taking the three milk products into consideration, HI was used to estimate the total health risk caused by multiple foodstuffs. The average HI calculated on the basis of average consumption rates for differently aged people were $2.424,0.310,0.105,0.070,0.067$, and 0.057 for groups of age $\leq 3,3<$ age $\leq 14,14<$ age $\leq 20,20<$ age $\leq 40,40<$ age $\leq 60$, and age $>60$, respectively (Table 6 ). It is noticed that the $\mathrm{HI}$ value of the maximum exposures for the 0-3 age group were high and up to 5.144. The data of $\mathrm{HI}$ suggests that kids under 3 years old are already experiencing adverse health effects on the basis of their current intake rate. Although $\mathrm{HI}$ value for teenagers (3-14 years) was within the safe level $(\mathrm{HI}<1)$, the potential health risk for teenagers is relatively high on comparison with that for adults in terms of HIs. Similar results have been reported by earlier studies [19, 31]. Consequently, it will be of great importance for children to control the dietary intake rate of milk products, especially kids under 3 year old.

\section{Conclusion}

Contents of metals in milk products (fermented, sterilized, and modified milk) in Nanchang, China, were measured in the present study. The overall mean concentrations of metals were in the order: $\mathrm{Zn}>\mathrm{Al}>\mathrm{Cu}>\mathrm{Ni}>\mathrm{Cr}>\mathrm{Pb}>$ $\mathrm{Co}>\mathrm{Cd}$, and the contents of $\mathrm{Zn}$ were significantly higher than other elements. It was observed that metals in milk products were all below the safety concentration limits, with the exception of $\mathrm{Zn}$ in fermented milk. The mean THQ values and TTHQ values for all age groups were below 1 . However, the TTHQ based on the maximum exposure level had values greater than one for kids under 3 years old. The HI values for kids of 0-3 age group were larger than one, suggesting that there was a potential health risk for these children. The estimation of health risks also indicated that Co was the largest contributor to TTHQ (followed by $\mathrm{Zn}$ ), and fermented milk was the major source of health risk in the three milk products. The results demonstrated that the consumption of fermented, sterilized, or modified milk on average will not result in a significant health risk for general people, but it is suggested that teenagers avoid drinking too much milk and milk products in the region, especially for ages 0-3 age.

\section{Acknowledgements}

This work is supported by a project founded by the Priority Academic Program Development of Jiangsu Higher Education Institute (PAPD) and the Scientific Research Program of Jiangxi (2013BBG70098). 


\section{References}

1. BULDinI P.L., CAVALLI S., SHARMA J.L. Matrix removal for the ion chromatographic determination of some trace elements in milk. Microchem J. 72, (3), 277, 2002.

2. SOMA G., GURDEEP S., JHA V.N., TRIPATHI R.M. Risk assessment due to ingestion of natural radionuclides and heavy metals in the milk samples: a case study from a proposed uranium mining area, Jharkhand. Environ. Monit. Assess. 175, (1-4), 157, 2011.

3. LICATA P., TROMBETTA D., CRISTANI M., GIOFRE F., MARTINO D., CALO M., NACCARI F. Levels of "toxic" and "essential" metals in samples of bovine milk from various dairy farms in Calabria, Italy. Environ. Int. 30, (1), 1, 2004.

4. MALHAT F., HAGAG M., SABER A., FAYZ A.E. Contamination of cows milk by heavy metal in Egypt. B. Environ. Contam. Tox. 88, (4), 611, 2012.

5. TONA G.O., ADETUNJI V.O., AMEEN S.A., IBIKUNLE A.O. Evaluation of Lead and Cadmium heavy metal residues in milk and milk products sold in Ogbomoso, Southwestern Nigeria. Pakistan Journal of Nutrition. 12, (2), 168, 2013

6. PILARCZYK R., WÓJCIK J., CZERNIAK P., SABLIK P., PILARCZYK B., TOMZA-MARCINIAK A. Concentration of toxic heavy metals and trace elements in raw milk of Simmental and Holstein-Friesian cows from organic farm. Environ. Monit. Assess. 185, (10), 8383, 2013.

7. KHAN K., LU Y., ISHTIAQ M., KHAN S., WAQAS M., WEI L., WANG T. Heavy metals in agricultural soils and crops and their health risks in Swat District, northern Pakistan. Food Chem. Toxicol. 58, 449, 2013.

8. JARUP L. Hazards of heavy metal contamination. Brit. Med. Bull. 68, 167, 2003.

9. CAO H.B., ZHANG H., QIAO L., CHEN J.J. Exposure and risk assessment for aluminium and heavy metals in Puerh tea. Sci. Total Environ. 408, (14), 2777, 2010.

10. POWERS K.M., SMITH W.T., FRANKLIN G.M., LONGSTRETH W.J., SWANSON P., CHECKOWAY H. Parkinson's disease risks associated with dietary iron, manganese, and other nutrient intakes. Neurology. 60, (11), 1761, 2003.

11. FU Q.L., LIU Y.L., LI L.H., ACHAL V. A survey on the heavy metal contents in Chinese traditional egg products and their potential health risk assessment. Food Additive \& Contaminants: Part B: Surveillance. 7, (2), 99, 2014.

12. FU Q.L., LI L.H., ACHAL V., JIAO A.Y., LIU Y.L. Concentrations of heavy metals and Arsenic in market rice grain and their potential health risks to the population of Fuzhou, China. Human and Ecological Risk Assessment: An International Journal. 21, (1), 117, 2015.

13. SAC (Standardization Administration of the People's Republic of China). Hygienic standard for foodstuffs (GB 2762-2012). Beijing: Chinese Standard Publishing House. 2012.

14. HUANG Z., PAN X.D., PING G., HAN J.L., CHEN Q. Health risk assessment of heavy metals in rice to the population in Zhejiang, China. PLoS One. 8, (9), 1, 2013.

15. US EPA. Guidance for Performing Aggregate Exposure and Risk Assessments. Washington DC: U.S. Environmental Protection Agency, Office of Pesticide Programs; 1999.
16. JECFA. Summary and Conclusions of the $61^{\text {st }}$ Meeting of the Joint FAO/WHO Expert Committee on Food Additives. JECFA/61/Sc Rome, Italy. 2003.

17. US EPA. Regional Screening Level (RSL) Summary Table-May 2014.

http://www.epa.gov/reg3hwmd/risk/human/rb-concentration table/Generic_Tables/docs/master_sl table_01run_MAY2 014.pdf. Accessed on 13 July, 2014.

18. ZOU X.J., QIU R.L., ZHOU X.Y., ZHENG W.H. Heavy metal contamination and health risk assessment in Dabao Mountain, China. Acta Scientiae Circumstantiae, 28, (7), 1406, 2008.

19. YANG M., JIANG L.X., HUANG H.P., SHENG B., QIU F., YU M., LI X.R., WEI S. Dietary exposure to Aluminium and health risk assessment in the residents of Shenzhen, China. PLoS ONE. 9, (3), 1, 2014.

20. SAHA N., ZAMAN M.R. Evaluation of possible health risks of heavy metals by consumption of foodstuffs available in the central market of Rajshahi City, Bangladesh. Environ. Monit. Assess. 185, (5), 3867, 2013.

21. US EPA. Guidelines for the health risk assessment of chemical mixtures. Fed Regist. 51, 34014, 1986.

22. JAVED I., JAN I., MUHAMMAD F., ZIA U.R., KHAN M.Z., ASLAM B., SULTAN J.I. Heavy metal residues in the milk of cattle and goats during winter season. B. Environ. Contam. Tox. 82, (5), 616, 2009.

23. DOBRZANSKI Z., KOLACZ R., GORECKA H., CHOJNACKA K., BARTKOWIAK A. The content of microelements and trace elements in raw milk from cows in the Silesian Region. Pol. J. Environ. Stud. 14, (5), 685, 2005.

24. IDF. International Dairy Federation Bulletin, Chemical Residues in milk and milk products. I.D.F. Document, 133, 1979.

25. MOHAMMAD R., HAJAR A.D., HASSAN J., ALI F., ARMAN S., HOSSEIN J., HOSSEIN T., MOHAMAD Y., ALI A.M. Assessment of dairy products consumed on the Arak market as determined by heavy metal residues. Health. 6, (5), 323, 2014

26. ENB A., ABOU DONI M.A., ABD-RABOU N.S., ABOUARAB A.A.K., EL-SENAITY M.H. Chemical composition of raw milk and heavy metals behavior during processing of milk products. Global Veterinaria. 3, (3), 268, 2009.

27. LANTE A., LOMOLINO G., CAGNIN M., SPETTOLI P. Content and characterization of minerals in milk and in Crescenza and Squacquerone Italian fresh cheeses by ICPOES. Food Control. 17, 299, 2006.

28. TRIPATHI R.M., RAGHUNATH R., SASTRY V.N., KRISHNAMOORTHY T.M. Daily intake of heavy metals by infants through milk and milk products. Sci. Total Environ. 277, (2), 299, 1999.

29. ZHUANG P., MCBRIDE M.B., XIA H., LI N., LI Z. Health risk from heavy metals via consumption of food crops in the vicinity of Dabaoshan mine, South China. Sci. Total Environ. 407, (5), 1551, 2009.

30. ZHANG P., HE Z.Y., LIANG G.D. Health risk assessment of contaminants in milk in Wuhan country. Food Science. 7, 417, 2007.

31. MAYURI C., ARVIND K.N. Heavy metals assessment in urban soil around industrial clusters in Ghaziabad, India: Probabilistic health risk approach. Ecotox. Environ. Safe. 87, 57, 2013. 